\title{
Synthesis of Some Novel Fused Imidazo $[2,1-b][1,3]$ Thiazole and Imidazo $[2,1-b]$ Thiazolo [5, 4-d] Isoxazole Derivatives
}

\author{
HAMID REZA JABERI and HADI NOORIZADEH* \\ Department of Chemistry, Faculty of Sciences \\ Ilam Branch, Islamic Azad University, Ilam Iran \\ Hadinoorizadeh@yahoo.com
}

Received 26 September 2011; Accepted 13 November 2011

\begin{abstract}
In this work we describe the synthesis of some novel fused imidazo $[2,1-b][1,3]$ thiazole derivatives. The reaction of 1,2 -diaminoethane 1 with carbon disulphide in $\mathrm{H}_{2} \mathrm{O} / \mathrm{ETOH}$ as solvent furnishes 4, 5-dihydro- $1 \mathrm{H}$ imidazol-2-thiol 2 under reflux condition. the reaction of 4,5-dihydro- $1 \mathrm{H}$ imidazol-2-thiol on treatment with ethylchloro acetate and aromatic aldehyde in presence of anhydrous sodium acetate and acetic acid as solvent to give (Z)2-(arylidene)-5,6-dihydroimidazo [2,1-b] [1,3] thiazol-3(2H)-one 3a-j. Compounds 3a-j was condensed with hydroxylamine to give 3-(aryl)-2, 3, 6, 7-tetrahydroimidazo [2, 1-b] [1,3] thiazolo [5, 4-d] isoxazole 4a-j. The structures of the new compounds were established by elemental analyses, IR, ${ }^{1} \mathrm{H}$ NMR and ${ }^{13} \mathrm{C}$ NMR data.
\end{abstract}

Keyword: Imidazole, Condensation, Synthesis, Imidazo [2, 1-b] [1, 3] thiazole, Heterocyclic compounds.

\section{Introduction}

In the family of heterocyclic compounds, nitrogen and sulfur containing heterocyles are an important class of compounds in medicinal chemistry ${ }_{1}$. Thus, fused heterocyclic derivatives with thiazole moiety are prospective objects in modern drug discovery. Various biologically active synthetic compounds have five-membered nitrogen-containing heterocyclic ring in their structures ${ }^{2}$. Structural frameworks have been described as privileged structures and in particular, N-containing polycyclic structures have been reported to be associated with a wide range of biological activity. In the field of five membered heterocyclic structures imidazole nucleus shows various properties. The high therapeutic properties of the imidazole related drugs have encouraged the medicinal chemists to synthesize a large number of novel chemotherapeutic agents. Imidazole drugs have broadened scope in remedying various dispositions in clinical medicines. A lot of work on the synthesis and biological activities of the condensed imidazo $[2,1-b]$-thiazoles has been reported. The imidazo $[2,1-b][1,3]$ thiazole skeleton has been used as anthelmintic agents, anti-hypertensives, anti-inflammatories, immunosuppressive agents, fungicides, herbicides, antitumor agents and cardiotonic agents ${ }^{3-14}$. 
Considering the potent bioactivities of compounds possessing an imidazothiazole core, synthesizing new imidazo $[2,1-b][1,3]$ thiazole derivatives efficiently attracted our attention ${ }^{15-18}$. In this paper we wish to report the synthesis of some novel fused imidazo [2, 1-b] [1, 3] thiazole derivatives that probably all new synthesized compounds have Pharmacology activities. The synthetic route is depicted in Figure 1. The structures of the compounds were supported by elemental and spectral data.

\section{Experimental}

Melting points were determined using an electro thermal digital apparatus and are uncorrected. IR spectra were recorded in film or in potassium bromide disks on a the Mattson Galaxy series FT-IR 5000 spectrophotometer $\left(v\right.$ max in $\mathrm{cm}^{-1}$ ) and NMR spectra were recorded on Bruker spectrophotometer $(300 \mathrm{MHz})$ in $\mathrm{DMSO}-\mathrm{d}_{6}$ or $\mathrm{CDCl}_{3}$ using TMS as an internal standard. Elemental analysis was performed on an Elemental Analyzer (Vario EL III). The progress of the reaction was monitored on a ready made silicagel plates (Merck) using n-hexane: ethyl acetate as a solvent system. Iodine was used as a developing agent. Spectral data (IR, NMR) confirmed the structures of the synthesized compounds and the purity of these compounds were ascertained by microanalysis. Elemental $(\mathrm{C}, \mathrm{H}, \mathrm{N})$ analysis indicated that the calculated and observed values were within the acceptable limits $( \pm 0.4 \%)$. Synthetic route is depicted in Figure 1.

\section{Synthesis of 4, 5-dihydro-1H-imidazol-2-thiol (2)}

To a solution of compound $1(0.2 \mathrm{~mol}, 13.37 \mathrm{~mL})$ in $\mathrm{H}_{2} \mathrm{O} / \mathrm{ETOH}(20 \mathrm{~mL}), \mathrm{CS}_{2}(0.2 \mathrm{~mol}$, $7.31 \mathrm{~mL})$ was added dropwise at $\left(0-5^{\circ} \mathrm{C}\right)$, the mixture was refluxed under stirring for about $12 \mathrm{~h}$. Then, the mixture was then cooled to room temperature and the crystalline product which separated was removed by filtration. The crude product was crystallized from ethanol to give the corresponding 4, 5-dihydro- $1 H$-imidazol-2-thiol as fine white needles.

\section{General procedure for the synthesis of compounds (3a-j)}

A mixture of compound $2(0.01 \mathrm{~mol}, 1.02 \mathrm{~g})$, ethylchloro acetate $(0.012 \mathrm{~mol}, 1.05 \mathrm{~mL})$ and anhydrous sodium acetate $(0.025 \mathrm{~mol}, 2 \mathrm{~g})$ in glacial acetic acid $(30 \mathrm{~mL})$, acetic anhydride $(10 \mathrm{~mL})$ mixture and aromatic aldehyde $(0.01 \mathrm{~mol})$ was refluxed for $5-7 \mathrm{~h}$, the reaction mixture was cooled and poured onto cold water $(100 \mathrm{~mL})$, the solid formed was filtered off and crystallized from appropriate solvent to give 3b-i. But for benzaldehyde and furfural (2-furaldehyde) derivatives the solution was concentrated by evaporation under reduced pressure. Then the solid was filtered off and crystallized from appropriate solvent to afford compounds $\mathbf{3 a}$ and $\mathbf{3 j}$.

General procedure for the synthesis of compounds (4a-j)

A mixture of compound 3a-j $(0.005 \mathrm{~mol})$, hydroxylamine hydrochloride $(0.005 \mathrm{~mol}, 0.04 \mathrm{~g})$ and anhydrous sodium acetate $(0.0125 \mathrm{~mol}, 1 \mathrm{~g})$ was refluxed in glacial acetic acid for $6-9 \mathrm{~h}$. The reaction mixture was allowed to cool and was poured into water $(100 \mathrm{~mL})$. The solid substance was filtered off and crystallized from appropriate solvent to give compound $\mathbf{4 a - j}$.

\section{4, 5-Dihydro-1H-imidazol-2-thiol (2)}

Yield 87\%, M.p. $196-200{ }^{\circ} \mathrm{C}$ IR $\left(\mathrm{KBr}, \mathrm{cm}^{-1}\right): v=3271(2 \mathrm{NH}), 1201(\mathrm{C}=\mathrm{S}) .{ }^{1} \mathrm{H}$ NMR $(300$

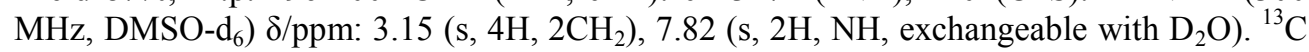
NMR (300 MHz, DMSO-d ${ }_{6}$ ) $/$ ppm: 43.22, 183.12 Anal.calcd for $\mathrm{C}_{3} \mathrm{H}_{6} \mathrm{~N}_{2} \mathrm{~S}: \mathrm{C}, 35.27 ; \mathrm{H}$, 5.92; N, 27.42; S, 31.39. Found: C, 35.20; H, 5.86; N, 27.45; S, 31.33 .

\section{(Z)-2-Benzylidene-5, 6-dihydroimidazo [2, 1-b] thiazol-3(2H)-one (3a)}

Yield 63\%, M.p. $143-145{ }^{\circ} \mathrm{C}$ IR $\left(\mathrm{KBr}, \mathrm{cm}^{-1}\right): v=3070$ (aromatic $\mathrm{C}-\mathrm{H}$ stretching), 1710 $\left(\mathrm{C}=\mathrm{O}\right.$ stretching), $1610(-\mathrm{C}=\mathrm{CH}$ stretching $), 1214(\mathrm{C}-\mathrm{N}) .{ }^{1} \mathrm{H}$ NMR (300 MHz, DMSO$\left.\mathrm{d}_{6}\right) \delta / \mathrm{ppm}: 3.72\left(\mathrm{t}, \mathrm{J}=5.25 \mathrm{~Hz}, 2 \mathrm{H}, \mathrm{CH}_{2}\right), 3.86\left(\mathrm{t}, \mathrm{J}=5.78 \mathrm{~Hz}, 2 \mathrm{H}, \mathrm{CH}_{2}\right), 7.45(\mathrm{~m}, 5 \mathrm{H}$, 
$\mathrm{H}_{\text {arom }}$ ), 7.76 (s, 1H, $\left.\mathrm{H}_{\text {methylidene }}\right) .{ }^{13} \mathrm{C}$ NMR (300 MHz, DMSO-d 6 ) $\delta / p p m: ~ 40.13,46.22$, $120.09,127.85,127.91,128.06,131.13,138.55,148.98,165.25$ Anal.calcd for $\mathrm{C}_{12} \mathrm{H}_{10} \mathrm{~N}_{2} \mathrm{OS}$ : C, 62.59; H, 4.38; N, 12.16; S, 13.92. Found: C, 62.50; H, 4.31; N, 12.11; S, 13.88 .

\section{(Z)-2-(2-Hydroxybenzylidene)-5, 6-dihydroimidazo [2, 1-b] thiazol-3(2H)-one (3b)}

Yield 58\%, M.p. $258-260{ }^{\circ} \mathrm{C}$ IR $\left(\mathrm{KBr}, \mathrm{cm}^{-1}\right): \mathrm{v}=3228$ (-OH stretching), 3062 (aromatic $\mathrm{C}-\mathrm{H}$ stretching), 2938 (aliphatic $\mathrm{C}-\mathrm{H}$ stretching), 1711 (C=O stretching), $1606(-\mathrm{C}=\mathrm{CH}$

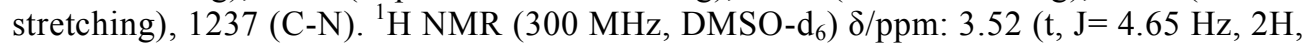
$\left.\mathrm{CH}_{2}\right), 3.70\left(\mathrm{t}, \mathrm{J}=5.28 \mathrm{~Hz}, 2 \mathrm{H}, \mathrm{CH}_{2}\right), 6.94-7.34\left(\mathrm{~m}, 4 \mathrm{H}, \mathrm{H}_{\text {arom }}\right), 7.91$ (s, $1 \mathrm{H}, \mathrm{H}_{\text {methylidene }}$ ), $9.56(\mathrm{bs}, 1 \mathrm{H}, \mathrm{OH}) .{ }^{13} \mathrm{C}$ NMR $\left(300 \mathrm{MHz}, \mathrm{DMSO}-\mathrm{d}_{6}\right) \delta / \mathrm{ppm}: 40.38,46.49,118.12$, $118.20,120.21,120.64,122.04,122.22,140.12,150.02,151.86,165.50$ Anal.calcd for $\mathrm{C}_{12} \mathrm{H}_{10} \mathrm{~N}_{2} \mathrm{O}_{2} \mathrm{~S}$ : C, 58.52; H, 4.09; N, 11.37; S, 13.02. Found: C, 58.44; H, 3.95; N, 11.28; $\mathrm{S}, 12.98$.

\section{(Z)-2-(4-Methylbenzylidene) - 5, 6-dihydroimidazo [2, 1-b] thiazol-3(2H)-one (3c)}

Yield 68\%, M.p. $165-167^{\circ} \mathrm{C}$ IR $\left(\mathrm{KBr}, \mathrm{cm}^{-1}\right)$ : $v=2998$ (aromatic C-H stretching), $1707(\mathrm{C}=\mathrm{O}$ stretching), $1619\left(-\mathrm{C}=\mathrm{CH}\right.$ stretching), $1216(\mathrm{C}-\mathrm{N}) .{ }^{1} \mathrm{H}$ NMR $\left(300 \mathrm{MHz}, \mathrm{CDCl}_{3}\right) \delta / \mathrm{ppm}: 2.37$ $\left(\mathrm{s}, 3 \mathrm{H}, \mathrm{CH}_{3}\right), 3.66\left(\mathrm{t}, \mathrm{J}=4.92 \mathrm{~Hz}, 2 \mathrm{H}, \mathrm{CH}_{2}\right), 3.77\left(\mathrm{t}, \mathrm{J}=5.73 \mathrm{~Hz}, 2 \mathrm{H}, \mathrm{CH}_{2}\right), 7.24(\mathrm{~d}, \mathrm{~J}=7.70$ $\left.\mathrm{Hz}, 2 \mathrm{H}, \mathrm{H}_{\text {arom }}\right), 7.36\left(\mathrm{~d}, \mathrm{~J}=7.80 \mathrm{~Hz}, 2 \mathrm{H}, \mathrm{H}_{\text {arom }}\right), 7.65\left(\mathrm{~s}, 1 \mathrm{H}, \mathrm{H}_{\text {methylidene }}\right) .{ }^{13} \mathrm{C}$ NMR $(300$ $\mathrm{MHz}, \mathrm{CDCl}_{3}$ ) $\delta$ /ppm: 21.46, 40.44, 46.80, 120.33, 129.60, 129.66, 129.79, 131.04, 139.83, 149.92, 165.15 Anal.calcd for $\mathrm{C}_{13} \mathrm{H}_{12} \mathrm{~N}_{2} \mathrm{OS}$ : C, 63.91; H, 4.95; N, 11.47; S, 13.12. Found: $\mathrm{C}$, 63.85; H, 4.89; N, 11.41; S, 13.07 .

\section{(Z)-2-(3-Methoxybenzylidene) - 5, 6-dihydroimidazo [2, 1-b] thiazol-3(2H)-one (3d)}

Yield 65\%, M.p. $137-139{ }^{\circ} \mathrm{C}$ IR $\left(\mathrm{KBr}, \mathrm{cm}^{-1}\right): v=3056$ (aromatic C-H stretching), 2933 (aliphatic C-H stretching), $1708\left(\mathrm{C}=\mathrm{O}\right.$ stretching), 1617 $\left(-\mathrm{C}=\mathrm{CH}\right.$ stretching), $1226(\mathrm{C}-\mathrm{N}) .{ }^{1} \mathrm{H}$

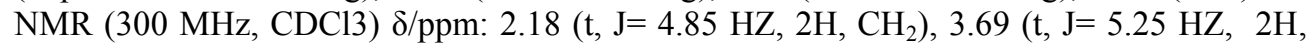
$\left.\mathrm{CH}_{2}\right), 3.82\left(\mathrm{~s}, 3 \mathrm{H}, \mathrm{OCH}_{3}\right), 6.94\left(\mathrm{~d}, \mathrm{~J}=7.84,1 \mathrm{H}, \mathrm{H}_{\text {arom }}\right), 7.02\left(\mathrm{~s}, 1 \mathrm{H}, \mathrm{H}_{\text {arom }}\right), 7.10(\mathrm{~d}, \mathrm{~J}=7.26$, $\left.1 \mathrm{H}, \mathrm{H}_{\text {arom }}\right), 7.37\left(\mathrm{t}, \mathrm{J}=7.78,1 \mathrm{H}, \mathrm{H}_{\text {arom }}\right), 7.68\left(\mathrm{~s}, 1 \mathrm{H}, \mathrm{H}_{\text {methylidene }}\right) .{ }^{13} \mathrm{C}$ NMR $(300 \mathrm{MHz}$, CDCl3) $\delta /$ ppm: 40.19, 46.22, 57.33, 117.06, 118.42, 120.16, 120.78, 131.22, 132.63, 139.56, 149.17, 160.18, 165.28 Anal.calcd for $\mathrm{C}_{13} \mathrm{H}_{12} \mathrm{~N}_{2} \mathrm{O}_{2} \mathrm{~S}: \mathrm{C}, 59.98 ; \mathrm{H}, 4.65 ; \mathrm{N}, 10.76 ; \mathrm{S}, 12.32$. Found: C, 59.92; H, 4.62; N, 10.69; S, 12.29 .

\section{(Z)-2-(3-Bromobenzylidene) - 5, 6-dihydroimidazo [2, 1-b] thiazol-3(2H)-one (3e)}

Yield 56\%, M.p. $170-172{ }^{\circ} \mathrm{C}$ IR $\left(\mathrm{KBr}, \mathrm{cm}^{-1}\right): \mathrm{v}=3057$ (aromatic C-H stretching), 2957 (aliphatic C-H stretching), $1702\left(\mathrm{C}=\mathrm{O}\right.$ stretching), $1643\left(-\mathrm{C}=\mathrm{CH}\right.$ stretching), $1226(\mathrm{C}-\mathrm{N}) .{ }^{1} \mathrm{H}$

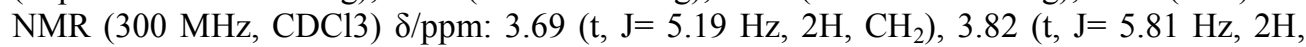
$\left.\mathrm{CH}_{2}\right), 7.32\left(\mathrm{t}, \mathrm{J}=7.76 \mathrm{~Hz}, 1 \mathrm{H}, \mathrm{H}_{\text {arom }}\right), 7.41\left(\mathrm{~d}, \mathrm{~J}=7.57 \mathrm{~Hz}, 1 \mathrm{H}, \mathrm{H}_{\text {arom }}\right), 7.49(\mathrm{~d}, \mathrm{~J}=7.68 \mathrm{~Hz}$, $\left.1 \mathrm{H}, \mathrm{H}_{\text {arom }}\right), 7.59\left(\mathrm{~d}, 2 \mathrm{H}, \mathrm{H}_{\text {arom }}+\mathrm{H}_{\text {methylidene }}\right) .{ }^{13} \mathrm{C} \mathrm{NMR}(300 \mathrm{MHz}, \mathrm{CDCl} 3) \delta / \mathrm{ppm}: 40.23$, $45.89,119.95,127.22,128.15,128.38,129.53,130.84,133.24,140.08,150.10,165.11$ Anal.calcd for $\mathrm{C}_{12} \mathrm{H}_{9} \mathrm{~N}_{2} \mathrm{OSBr}$ : C, 46.62; H, 2.93; N, 9.06; S, 10.37.Found: C, 46.66; H, 2.87; $\mathrm{N}, 8.98 ; \mathrm{S}, 10.34$.

(Z)-2-(3-Chlorobenzylidene) - 5, 6-dihydroimidazo [2, 1-b] thiazol-3(2H)-one (3f)

Yield 54\%, M.p. $161-162{ }^{\circ} \mathrm{C}$ IR $\left(\mathrm{KBr}, \mathrm{cm}^{-1}\right): \mathrm{v}=3050$ (aromatic C-H stretching), 2955 (aliphatic C-H stretching), $1698\left(\mathrm{C}=\mathrm{O}\right.$ stretching), $1632\left(-\mathrm{C}=\mathrm{CH}\right.$ stretching), $1219(\mathrm{C}-\mathrm{N}) .{ }^{1} \mathrm{H}$

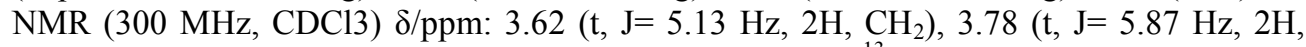
$\left.\mathrm{CH}_{2}\right), 7.37-7.52\left(\mathrm{~m}, 4 \mathrm{H}, \mathrm{H}_{\text {arom }}\right), 7.56\left(\mathrm{~s}, 1 \mathrm{H}, \mathrm{H}_{\text {methylidene }}\right) .{ }^{13} \mathrm{C} \mathrm{NMR}(300 \mathrm{MHz}, \mathrm{CDCl} 3)$

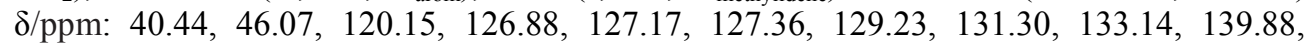


150.23, 165.32 Anal.calcd for $\mathrm{C}_{12} \mathrm{H}_{9} \mathrm{~N}_{2} \mathrm{OSCl}$ : C, 46.62; H, 2.93; N, 9.06; S, 10.37.Found: $\mathrm{C}$, 46.57; H, 2.86; N, 9.11; S, 10.32 .

\section{(Z)-2-(2-Nitrobenzylidene) - 5, 6-dihydroimidazo [2, 1-b] thiazol-3(2H)-one (3g)}

Yield 55\%, M.p. $114-116{ }^{\circ} \mathrm{C}$ IR $\left(\mathrm{KBr}, \mathrm{cm}^{-1}\right): \mathrm{v}=3048$ (aromatic C-H stretching), 2987 (aliphatic $\mathrm{C}-\mathrm{H}$ stretching), $1713\left(\mathrm{C}=\mathrm{O}\right.$ stretching), $1623(-\mathrm{C}=\mathrm{CH}$ stretching $), 1524\left(\mathrm{NO}_{2}\right.$ asym), 1231(C-N). ${ }^{1} \mathrm{H}$ NMR (300 MHz, $\mathrm{CDCl}_{3}$ ) $\delta / \mathrm{ppm}: 3.70$ (t, J=4.95 Hz, 2H, $\mathrm{CH}_{2}$ ), 3.87 $\left(\mathrm{t}, \mathrm{J}=5.50 \mathrm{~Hz}, 2 \mathrm{H}, \mathrm{CH}_{2}\right), 7.57\left(\mathrm{t}, \mathrm{J}=7.35 \mathrm{~Hz}, 1 \mathrm{H}, \mathrm{H}_{\text {aromat }}\right), 7.71\left(\mathrm{~m}, 2 \mathrm{H}, \mathrm{H}_{\text {arom }}\right), 8.07(\mathrm{~s}, 1 \mathrm{H}$, $\left.\mathrm{H}_{\text {methylidene }}\right), 8.14\left(\mathrm{~d}, \mathrm{~J}=8.04 \mathrm{~Hz}, 1 \mathrm{H}, \mathrm{H}_{\text {arom }}\right) .{ }^{13} \mathrm{C} \mathrm{NMR}\left(300 \mathrm{MHz}, \mathrm{CDCl}_{3}\right) \delta / \mathrm{ppm}: 40.80$, $46.75,120.28,125.70,127.30,127.41,129.63,131.43,139.10,141.18,150.05,165.42$ Anal.calcd for $\mathrm{C}_{12} \mathrm{H}_{9} \mathrm{~N}_{3} \mathrm{O}_{3} \mathrm{~S}$ : C, 52.36; H, 3.30; N, 15.26; S, 11.65. Found: C, $52.29 \mathrm{H}, 3.26$; $\mathrm{N}, 15.21 ; \mathrm{S}, 11.61$.

\section{(Z)-2-(3-nitrobenzylidene) - 5, 6-dihydroimidazo [2, 1-b] thiazol-3(2H)-one (3h)}

Yield 60\%, M.p. $199-201^{\circ} \mathrm{C}$ IR $\left(\mathrm{KBr}, \mathrm{cm}^{-1}\right): \mathrm{v}=3061$ (aromatic C-H stretching), $1709(\mathrm{C}=\mathrm{O}$ stretching), $1614\left(-\mathrm{C}=\mathrm{CH}\right.$ stretching), $1515\left(\mathrm{NO}_{2}\right.$ asym), $1225(\mathrm{C}-\mathrm{N}) .{ }^{1} \mathrm{H}$ NMR $(300 \mathrm{MHz}$,

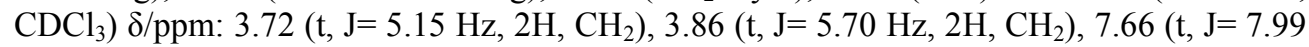
$\left.\mathrm{Hz}, 1 \mathrm{H}, \mathrm{H}_{\text {arom }}\right), 7.72\left(\mathrm{~s}, 1 \mathrm{H}, \mathrm{H}_{\text {methylidene }}\right), 7.81\left(\mathrm{~d}, \mathrm{~J}=7.49 \mathrm{~Hz}, 1 \mathrm{H}, \mathrm{H}_{\text {arom }}\right), 8.23$ (d, J= 7.86 Hz $\left.1 \mathrm{H}, \mathrm{H}_{\text {arom }}\right), 8.35$ (s, $\left.1 \mathrm{H}, \mathrm{H}_{\text {arom }}\right) .{ }^{13} \mathrm{C} \mathrm{NMR}\left(300 \mathrm{MHz}, \mathrm{CDCl}_{3}\right) \delta / \mathrm{ppm}: 41.06,46.13,120.36$, $121.12,122.55,124.42,129.62,132.43,138.82,142.25,149.22,165.37$ Anal.calcd for $\mathrm{C}_{12} \mathrm{H}_{9} \mathrm{~N}_{3} \mathrm{O}_{3} \mathrm{~S}$ : C, 52.36; H, 3.30; N, 15.26; S, 11.65. Found: C, 52.31; H, 3.33; N, 15.21; S, 11.60 .

\section{(Z)-2-(4-Nitrobenzylidene) - 5, 6-dihydroimidazo [2, 1-b] thiazol-3(2H)-one (3i)}

Yield 58\%, M.p. $228-230{ }^{\circ} \mathrm{C}$ IR $\left(\mathrm{KBr}, \mathrm{cm}^{-1}\right): v=3050$ (aromatic C-H stretching), 2973 (aliphatic C-H stretching), $1712\left(\mathrm{C}=\mathrm{O}\right.$ stretching), $1605\left(-\mathrm{C}=\mathrm{CH}\right.$ stretching), $1505\left(\mathrm{NO}_{2}\right.$ asym), $1227(\mathrm{C}-\mathrm{N}) .{ }^{1} \mathrm{H}$ NMR $\left(300 \mathrm{MHz}, \mathrm{CDCl}_{3}\right) \delta / \mathrm{ppm}: 3.73\left(\mathrm{t}, \mathrm{J}=5.25 \mathrm{~Hz}, 2 \mathrm{H}, \mathrm{CH}_{2}\right)$ $3.86\left(\mathrm{t}, \mathrm{J}=5.81 \mathrm{~Hz}, 2 \mathrm{H}, \mathrm{CH}_{2}\right), 7.65$ (d, 2H, J=8.57 Hz, $\mathrm{H}_{\text {arom }}$ ), 7.73 (s, 1 H, $\mathrm{H}_{\text {methylidene }}$ ), $8.32\left(\mathrm{~d}, \mathrm{~J}=8.52 \mathrm{~Hz}, 2 \mathrm{H}, \mathrm{H}_{\text {arom }}\right) .{ }^{13} \mathrm{C} \mathrm{NMR}\left(300 \mathrm{MHz}, \mathrm{CDCl}_{3}\right) \delta / \mathrm{ppm}: 40.24,46.38$, $120.14,126.25,126.34,132.74,139.83,145.73,149.50,165.31$ Anal.calcd for $\mathrm{C}_{12} \mathrm{H}_{9} \mathrm{~N}_{3} \mathrm{O}_{3} \mathrm{~S}$ : C, 52.36; H, 3.30; N, 15.26; S, 11.65. Found: C, 52.39; H, 3.26; N, 15.29; $\mathrm{S}, 11.59$.

\section{(Z)-2-(Furan-2-ylmethylene) - 5, 6-dihydroimidazo [2, 1-b] thiazol-3(2H)-one (3j)}

Yield 56\%, M.p. $145-147{ }^{\circ} \mathrm{C}$ IR $\left(\mathrm{KBr}, \mathrm{cm}^{-1}\right) v=2982$ (aromatic C-H stretching), 1709 $(\mathrm{C}=\mathrm{O}$ stretching $), 1618(-\mathrm{C}=\mathrm{CH}$ stretching $), 1231(\mathrm{C}-\mathrm{N}) .{ }^{1} \mathrm{H}$ NMR $\left(300 \mathrm{MHz}, \mathrm{DMSO}-\mathrm{d}_{6}\right)$

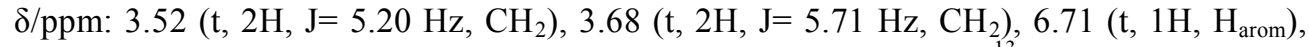
6.97 (d, $1 \mathrm{H}, \mathrm{H}_{\text {arom }}$ ), 7.48 (s, 1H, $\left.\mathrm{H}_{\text {methylidene }}\right), 8.00$ (d, 1H, $\left.\mathrm{H}_{\text {arom }}\right) .{ }^{13} \mathrm{C} \mathrm{NMR}(300 \mathrm{MHz}$, DMSO-d $\left.{ }_{6}\right) \delta /$ ppm: 40.32, 45.95, 116.08, 118.11, 122.26, 139.71, 144.44, 149.25, 150.51, 165.11 Anal.calcd for $\mathrm{C}_{10} \mathrm{H}_{8} \mathrm{~N}_{2} \mathrm{O}_{2} \mathrm{~S}: \mathrm{C}, 54.53 ; \mathrm{H}, 3.66 ; \mathrm{N}, 12.72 ; \mathrm{S}, 14.56$. Found: $\mathrm{C}$, 54.48; H, 3.69; N, 12.67; S, 14.51.

\section{3-Phenyl-2, 3, 6, 7-tetrahydroimidazo [2, 1-b] thiazolo [5, 4-d] isoxazole (4a)}

Yield 62\%, M.p. $162-164{ }^{\circ} \mathrm{C}$ IR $\left(\mathrm{KBr}, \mathrm{cm}^{-1}\right): v=3170(\mathrm{NH}), 3024$ (aromatic C-H stretching), 2985 (aliphatic C-H stretching), 1218(C-N). ${ }^{1} \mathrm{H}$ NMR (300 MHz, DMSO-d6) $\delta /$ ppm: 3.62 (t, J= $\left.5.35 \mathrm{~Hz}, 2 \mathrm{H}, \mathrm{CH}_{2}\right), 3.81$ (t, J=5.97 Hz, $\left.2 \mathrm{H}, \mathrm{CH}_{2}\right), 4.67\left(\mathrm{~s}, 1 \mathrm{H}, \mathrm{C}_{3}-\mathrm{H}\right), 7.38\left(\mathrm{~m}, 5 \mathrm{H}, \mathrm{H}_{\text {arom }}\right), 10.45$ (s, $1 \mathrm{H}, \mathrm{NH}$, exchangeable with $\mathrm{D}_{2} \mathrm{O}$ ). ${ }^{13} \mathrm{C}$ NMR (300 MHz, DMSO-d6) $\delta / \mathrm{ppm}: 41.73,46.91$, $71.18,96.12,127.10,127.26,127.65,131.27,150.42,169.12$ Anal.calcd for $\mathrm{C}_{12} \mathrm{H}_{11} \mathrm{~N}_{3} \mathrm{OS}$ : C, 58.76; H, 4.52; N, 17.13; S, 13.07.Found: C, 58.68; H, 4.47; N, 17.07; S, 13.12. 
3-(2-Hydroxyphenyl)-2, 3, 6, 7-tetrahydroimidazo [2, 1-b] thiazolo [5, 4-d] isoxazole (4b)

Yield 53\%, M.p. $271-273{ }^{\circ} \mathrm{C}$ IR $\left(\mathrm{KBr}, \mathrm{cm}^{-1}\right)$ : v= $3320(-\mathrm{OH}), 3184$ (NH), 3064 (aromatic CH stretching), 1219 (C-N). ${ }^{1} \mathrm{H}$ NMR (300 MHz, DMSO-d6) $\delta / \mathrm{ppm}: 3.49$ (t, J= $4.85 \mathrm{~Hz}, 2 \mathrm{H}$, $\left.\mathrm{CH}_{2}\right), 3.68\left(\mathrm{t}, \mathrm{J}=5.47 \mathrm{~Hz}, 2 \mathrm{H}, \mathrm{CH}_{2}\right), 4.71\left(\mathrm{~s}, 1 \mathrm{H}, \mathrm{C}_{3}-\mathrm{H}\right), 7.11-7.39$ (m, $\left.4 \mathrm{H}, \mathrm{H}_{\text {arom }}\right), 9.49$ (bs, $1 \mathrm{H}, \mathrm{OH}), 10.41\left(\mathrm{~s}, 1 \mathrm{H}, \mathrm{NH}\right.$, exchangeable with $\left.\mathrm{D}_{2} \mathrm{O}\right) .{ }^{13} \mathrm{C}$ NMR (300 MHz, DMSO-d6) $\delta /$ ppm: 42.09, 46.89, 66.58, 95.76, 118.66, 120.83, 121.05, 125.55, 125.91, 149.78, 151.13, 170.02 Anal.calcd for $\mathrm{C}_{12} \mathrm{H}_{11} \mathrm{~N}_{3} \mathrm{O}_{2} \mathrm{~S}: \mathrm{C}, 55.16 ; \mathrm{H}, 4.24 ; \mathrm{N}, 16.08 ; \mathrm{S}, 12.27$.Found: C, 55.09; $\mathrm{H}, 4.27 ; \mathrm{N}, 15.98 ; \mathrm{S}, 12.22$.

3-(4-Methylphenyl)-2, 3, 6, 7-tetrahydroimidazo [2, 1-b] thiazolo [5, 4-d] isoxazole (4c)

Yield 57\%, M.p. $180-182{ }^{\circ} \mathrm{C}$ IR $\left(\mathrm{KBr}, \mathrm{cm}^{-1}\right): v=3165(\mathrm{NH}), 3045$ (aromatic C-H stretching), 1229 (C-N). ${ }^{1} \mathrm{H}$ NMR $(300 \mathrm{MHz}, \mathrm{CDCl} 3) \delta / \mathrm{ppm}: 2.57$ (s, 3H, $\mathrm{CH}_{3}$ ), 3.68 (t, J= $\left.5.10 \mathrm{~Hz}, 2 \mathrm{H}, \mathrm{CH}_{2}\right), 3.79\left(\mathrm{t}, \mathrm{J}=5.85 \mathrm{~Hz}, 2 \mathrm{H}, \mathrm{CH}_{2}\right), 4.65\left(\mathrm{~s}, 1 \mathrm{H}, \mathrm{C}_{3}-\mathrm{H}\right), 7.10(\mathrm{~d}, \mathrm{~J}=7.65 \mathrm{~Hz}$, $\left.2 \mathrm{H}, \mathrm{H}_{\text {arom }}\right), 7.26\left(\mathrm{~d}, \mathrm{~J}=7.70 \mathrm{~Hz}, 2 \mathrm{H}, \mathrm{H}_{\text {arom }}\right), 10.45\left(\mathrm{~s}, 1 \mathrm{H}, \mathrm{NH}\right.$, exchangeable with $\left.\mathrm{D}_{2} \mathrm{O}\right) \cdot{ }^{13} \mathrm{C}$ NMR (300 MHz, CDCl3) $\delta / \mathrm{ppm}: 21.35,42.21,46.71,67.83,95.63,127.70,127.81,128.13$, 130.70, 149.85, 169.91 Anal.calcd for $\mathrm{C}_{13} \mathrm{H}_{13} \mathrm{~N}_{3} \mathrm{OS}: \mathrm{C}, 60.21 ; \mathrm{H}, 5.05 ; \mathrm{N}, 16.20 ; \mathrm{S}$, 12.36.Found: C, 60.17; H, 4. 98; N, 16.15; S, 12.31 .

3-(3-Methoxyphenyl)-2, 3, 6, 7-tetrahydroimidazo [2, 1-b] thiazolo [5, 4-d] isoxazole (4d)

Yield 52\%, M.p. $151-152{ }^{\circ} \mathrm{C}$ IR $\left(\mathrm{KBr}, \mathrm{cm}^{-1}\right): v=3154(\mathrm{NH}), 3014$ (aromatic C-H stretching), 2933 (aliphatic C-H stretching), 1217 (C-N). ${ }^{1} \mathrm{H}$ NMR (300 MHz, CDCl3) $\delta /$ ppm: $2.41\left(\mathrm{t}, \mathrm{J}=4.70 \mathrm{~Hz}, 2 \mathrm{H}, \mathrm{CH}_{2}\right), 3.65\left(\mathrm{t}, \mathrm{J}=5.20 \mathrm{~Hz}, 2 \mathrm{H}, \mathrm{CH}_{2}\right), 3.75\left(\mathrm{~s}, 3 \mathrm{H}, \mathrm{OCH}_{3}\right)$, $4.55\left(\mathrm{~s}, 1 \mathrm{H}, \mathrm{C}_{3}-\mathrm{H}\right), 6.75-6.89\left(\mathrm{~m}, 3 \mathrm{H}, \mathrm{H}_{\text {arom }}\right), 7.15\left(\mathrm{t}, \mathrm{J}=7.55 \mathrm{~Hz}, 1 \mathrm{H}, \mathrm{H}_{\text {arom }}\right), 10.38(\mathrm{~s}, 1 \mathrm{H}$, $\mathrm{NH}$, exchangeable with $\left.\mathrm{D}_{2} \mathrm{O}\right) .{ }^{13} \mathrm{C}$ NMR (CDCl3) $\delta / \mathrm{ppm}: 42.85,46.73,56.32,70.12,96.22$, $116.88,117.13,119.14,128.13,131.24,149.93,152.23,170.05$ Anal.calcd for $\mathrm{C}_{13} \mathrm{H}_{13} \mathrm{~N}_{3} \mathrm{O}_{2} \mathrm{~S}: \mathrm{C}, 56.71 ; \mathrm{H}, 4.76 ; \mathrm{N}, 15.26$; $\mathrm{S}, 11.65$.Found: $\mathrm{C}, 56.65 ; \mathrm{H}, 4.79 ; \mathrm{N}, 15.21 ; \mathrm{S}$, 11.59 .

3-(3-Bromophenyl)-2, 3, 6, 7-tetrahydroimidazo [2, 1-b] thiazolo [5, 4-d] isoxazole (4e)

Yield 56\%, M.p. 193-195 ${ }^{\circ} \mathrm{C}$ IR $\left(\mathrm{KBr}, \mathrm{cm}^{-1}\right): v=3162(\mathrm{NH}), 3067$ (aromatic C-H stretching), 2936 (aliphatic C-H stretching), 1234(C-N). ${ }^{1} \mathrm{H}$ NMR (300 MHz, CDCl3) $\delta / \mathrm{ppm}: 3.62\left(\mathrm{t}, \mathrm{J}=5.21 \mathrm{~Hz}, 2 \mathrm{H}, \mathrm{CH}_{2}\right), 3.75\left(\mathrm{t}, \mathrm{J}=5.71 \mathrm{~Hz}, 2 \mathrm{H}, \mathrm{CH}_{2}\right), 4.61\left(\mathrm{~s}, 1 \mathrm{H}, \mathrm{C}_{3}-\mathrm{H}\right)$, 7.15-7.42 (m, 4H, $\left.\mathrm{H}_{\text {arom }}\right), 10.47\left(\mathrm{~s}, 1 \mathrm{H}, \mathrm{NH}\right.$, exchangeable with $\left.\mathrm{D}_{2} \mathrm{O}\right) .{ }^{13} \mathrm{C}$ NMR $(\mathrm{CDCl} 3)$ $\delta / \mathrm{ppm}: 42.67,46.65,68.17,96.38,125.22,126.10,127.28,129.53,130.04,138.71,149.68$, 171.03 Anal.calcd for $\mathrm{C}_{12} \mathrm{H}_{10} \mathrm{~N}_{3} \mathrm{OSBr}$ : C, 44.46; H, 3.11; N, 12.96; S, 9.89. Found: C, 44.39; H, 3.07; N, 12.91; S, 9.93.

3-(3-Chlorophenyl)-2, 3, 6, 7-tetrahydroimidazo [2, 1-b] thiazolo [5, 4-d] isoxazole (3f)

Yield 53\%, M.p. 183-185 ${ }^{\circ} \mathrm{C}$ IR $\left(\mathrm{KBr}, \mathrm{cm}^{-1}\right)$ : v= 3159 (NH), 3047 (aromatic C-H stretching), 1228 (C-N). ${ }^{1} \mathrm{H}$ NMR (300 MHz, CDCl3) $\delta / \mathrm{ppm}: 3.65$ (t, J=5.05 Hz, 2H, CH2), $3.73(\mathrm{t}, \mathrm{J}=5.64 \mathrm{~Hz}, 2 \mathrm{H}, \mathrm{CH} 2), 4.70\left(\mathrm{~s}, 1 \mathrm{H}, \mathrm{C}_{3}-\mathrm{H}\right), 7.23-7.49\left(\mathrm{~m}, 4 \mathrm{H}, \mathrm{H}_{\text {arom }}\right), 10.41(\mathrm{~s}, 1 \mathrm{H}$, $\mathrm{NH}$, exchangeable with $\mathrm{D}_{2} \mathrm{O}$ ). ${ }^{13} \mathrm{C}$ NMR (CDCl3) $\delta / \mathrm{ppm}: 42.74,46.69,70.15,96.09,126.15$, $126.53,126.78,128.98,130.12,135.68,149.16,170.58$ Anal.calcd for $\mathrm{C}_{12} \mathrm{H}_{10} \mathrm{~N}_{3} \mathrm{OSCl}$ : C, 51.52; H, 3.60; N, 15.02; S, 11.46. Found: C, 51.56; H, 3.55; N, 14.97; S, 11.41 . 
3-(2-Nitrophenyl)-2, 3, 6, 7-tetrahydroimidazo [2, 1-b] thiazolo [5, 4-d] isoxazole (4g)

Yield 55\%, M.p. $132-134{ }^{\circ} \mathrm{C}$ IR $\left(\mathrm{KBr}, \mathrm{cm}^{-1}\right): v=3168(\mathrm{NH}), 3014$ (aromatic C-H stretching), 1549 ( $\mathrm{NO}_{2}$ asym), $1213(\mathrm{C}-\mathrm{N}) .{ }^{1} \mathrm{H}$ NMR (300 MHz, CDCl3) $\delta / \mathrm{ppm}: 3.61$ $\left(\mathrm{t}, \mathrm{J}=4.83 \mathrm{~Hz}, 2 \mathrm{H}, \mathrm{CH}_{2}\right), 3.77\left(\mathrm{t}, \mathrm{J}=5.45 \mathrm{~Hz}, 2 \mathrm{H}, \mathrm{CH}_{2}\right), 4.63\left(\mathrm{~s}, 1 \mathrm{H}, \mathrm{C}_{3}-\mathrm{H}\right), 7.72-8.05(\mathrm{~m}$, $\left.4 \mathrm{H}, \mathrm{H}_{\text {arom }}\right), 10.38\left(\mathrm{~s}, 1 \mathrm{H}, \mathrm{NH}\right.$, exchangeable with $\left.\mathrm{D}_{2} \mathrm{O}\right) .{ }^{13} \mathrm{C}$ NMR (CDCl3) $\delta / \mathrm{ppm}: 42.07$, $46.81,65.83,97.23,125.14,126.23,126.36,130.21,132.21,137.39,150.02,171.11$ Anal.calcd for $\mathrm{C}_{12} \mathrm{H}_{10} \mathrm{~N}_{4} \mathrm{O}_{3} \mathrm{~S}$ : C, 49.65; H, 3.47; N, 19.30; S, 11.05. Found: C, 49.57; H, $3.39 ; \mathrm{N}, 19.23 ; \mathrm{S}, 10.98$.

\section{3-(3-Nitrophenyl)-2, 3, 6, 7-tetrahydroimidazo [2, 1-b] thiazolo [5, 4-d] isoxazole} (4h)

Yield $60 \%$, M.p. $214-216{ }^{\circ} \mathrm{C}$ IR $\left(\mathrm{KBr}, \mathrm{cm}^{-1}\right): v=3171(\mathrm{NH}), 3074$ (aromatic C-H stretching), 2947 (aliphatic C-H stretching), 1537 ( $\mathrm{NO}_{2}$ asym), 1216 (C-N). ${ }^{1} \mathrm{H}$ NMR (300 $\mathrm{MHz}, \mathrm{CDCl} 3) \delta / \mathrm{ppm}: 3.66\left(\mathrm{t}, \mathrm{J}=5.20 \mathrm{~Hz}, 2 \mathrm{H}, \mathrm{CH}_{2}\right), 3.74\left(\mathrm{t}, \mathrm{J}=5.73 \mathrm{~Hz}, 2 \mathrm{H}, \mathrm{CH}_{2}\right), 4.58$ (s, $\left.1 \mathrm{H}, \mathrm{C}_{3}-\mathrm{H}\right), 7.57-7.69\left(\mathrm{~m}, 2 \mathrm{H}, \mathrm{H}_{\text {arom }}\right), 8.04\left(\mathrm{~d}, \mathrm{~J}=7.65 \mathrm{~Hz}, 1 \mathrm{H}, \mathrm{H}_{\text {arom }}\right), 8.21\left(\mathrm{~s}, 1 \mathrm{H}, \mathrm{H}_{\text {arom }}\right)$, $10.29\left(\mathrm{~s}, 1 \mathrm{H}, \mathrm{NH}\right.$, exchangeable with $\left.\mathrm{D}_{2} \mathrm{O}\right) \cdot{ }^{13} \mathrm{C}$ NMR $(\mathrm{CDCl} 3) \delta / \mathrm{ppm}: 42.61,46.64,66.12$, $98.89,123.03,124.18,126.68,129.28,130.89,144.26,150.23,169.92$ Anal.calcd for $\mathrm{C}_{12} \mathrm{H}_{10} \mathrm{~N}_{4} \mathrm{O}_{3} \mathrm{~S}: \mathrm{C}, 49.65 ; \mathrm{H}, 3.47 ; \mathrm{N}, 19.30 ; \mathrm{S}, 11.05$. Found: C, 49.60; H, 3.41; N, 19.25; S, 11.09 .

3-(4-Nitrophenyl)-2, 3, 6, 7-tetrahydroimidazo [2, 1-b] thiazolo [5, 4-d] isoxazole (4i)

Yield 63\%, M.p. $240-242{ }^{\circ} \mathrm{C}$ IR $\left(\mathrm{KBr}, \mathrm{cm}^{-1}\right): v=3168(\mathrm{NH}), 3012$ (aromatic C-H stretching), 1510 ( $\mathrm{NO}_{2}$ asym), $1222(\mathrm{C}-\mathrm{N}) .{ }^{1} \mathrm{H}$ NMR $(300 \mathrm{MHz}, \mathrm{CDCl} 3) \delta / \mathrm{ppm}: 3.66(\mathrm{t}, \mathrm{J}=$ $\left.5.15 \mathrm{~Hz}, 2 \mathrm{H}, \mathrm{CH}_{2}\right), 3.72\left(\mathrm{t}, \mathrm{J}=5.60 \mathrm{~Hz}, 2 \mathrm{H}, \mathrm{CH}_{2}\right), 4.61\left(\mathrm{~s}, 1 \mathrm{H}, \mathrm{C}_{3}-\mathrm{H}\right), 7.49(\mathrm{~d}, \mathrm{~J}=8.45 \mathrm{~Hz}$, $\left.2 \mathrm{H}, \mathrm{H}_{\text {arom }}\right), 7.89\left(\mathrm{~d}, \mathrm{~J}=8.40 \mathrm{~Hz}, 2 \mathrm{H}, \mathrm{H}_{\text {arom }}\right), 10.25\left(\mathrm{~s}, 1 \mathrm{H}, \mathrm{NH}\right.$, exchangeable with $\left.\mathrm{D}_{2} \mathrm{O}\right) \cdot{ }^{13} \mathrm{C}$ NMR (CDCl3) $\delta / \mathrm{ppm}$ : 42.53, 46.43, 68.11, 97.18, 126.08, 128.18, 141.26, 144.06, 149.73, 170.19 Anal.calcd for $\mathrm{C}_{12} \mathrm{H}_{10} \mathrm{~N}_{4} \mathrm{O}_{3} \mathrm{~S}: \mathrm{C}, 49.65 ; \mathrm{H}, 3.47 ; \mathrm{N}, 19.30 ; \mathrm{S}, 11.05$. Found: C, 49.69; $\mathrm{H}, 3.41 ; \mathrm{N}, 19.23 ; \mathrm{S}, 10.97$.

\section{3-(Furan-2-yl)-2, 3, 6, 7-tetrahydroimidazo [2, 1-b] thiazolo [5, 4-d] isoxazole (4j)}

Yield 51\%, M.p. $155-157{ }^{\circ} \mathrm{C}$ IR ( $\left.\mathrm{KBr}, \mathrm{cm}^{-1}\right) v=3173(\mathrm{NH}), 3019$ (aromatic C-H stretching), 2942 (aliphatic C-H stretching), 1237 (C-N). ${ }^{1} \mathrm{H}$ NMR ( $300 \mathrm{MHz}$, DMSO-d6) 8/ppm: 3.61 (t, J= $\left.5.07 \mathrm{~Hz}, 2 \mathrm{H}, \mathrm{CH}_{2}\right), 3.78\left(\mathrm{t}, \mathrm{J}=5.56 \mathrm{~Hz}, 2 \mathrm{H}, \mathrm{CH}_{2}\right), 4.64\left(\mathrm{~s}, 1 \mathrm{H}, \mathrm{C}_{3}-\mathrm{H}\right), 6.61-67.15\left(\mathrm{~m}, 3 \mathrm{H}, \mathrm{H}_{\text {arom }}\right)$, $10.37\left(\mathrm{~s}, 1 \mathrm{H}, \mathrm{NH}\right.$, exchangeable with $\left.\mathrm{D}_{2} \mathrm{O}\right){ }^{13} \mathrm{C}$ NMR (DMSO-d6) $\delta / \mathrm{ppm}: 41.89,46.13$, 69.02, 95.84, 112.21, 113.37, 139.88, 148.13, 150.42 170.21 Anal.calcd for $\mathrm{C}_{10} \mathrm{H}_{9} \mathrm{~N}_{3} \mathrm{O}_{2} \mathrm{~S}: \mathrm{C}$, 51.05; H, 3.86; N, 17.86; S, 13.63. Found: C, 50.97; H, 3.80; N, 17.79; S, 13.57 .

\section{Results and Discussion}

In this study we have prepared new imidazo [2, 1- b] [1, 3] thiazole derivatives from 4, 5-dihydro-1H-imidazol-2-thiol. The initial step in the synthetic method involved the synthesis of 4, 5-dihydro- $1 H$-imidazol-2-thiol 2 by refluxing 1, 2-diaminoethane and carbon disulphide. The synthesis route of compounds is outlined in Figure 1. In the second step, 4,5-dihydro- $1 H$-imidazol-2-thiol 2 was refluxed with ethyl chloroacetate and benzaldehyde derivatives in the presence of sodium acetate in acetic acid as solvent to give (Z)-2-(arylidene)- 5,6-dihydroimidazo [2, 1-b] [1,3] thiazol-3(2H)-one 3a-j, The presence of active methylene group in intermediate compound could be confirmed by condensation with benzaldehyde derivatives in the presence of sodium acetate in a mixture of glacial acetic acid/acetic anhydride to yield arylmethylene. The IR spectra of 
the latter compounds showed the presence of $\mathrm{C}=\mathrm{O}$ group. In the last step 3-(aryl)-2, 3, 6, 7-tetrahydroimidazo [2, 1-b] thiazolo [5, 4-d] isoxazole 4a-j were prepared separately from (Z)-2-(arylidene)- 5,6-dihydroimidazo [2, 1-b] [1,3] thiazol-3(2H)-one 3a-j and hydroxylamine hydrochloride in glacial acetic acid and anhydrous sodium acetate (Figure 1), according to reported methods ${ }^{15-18}$. No evidences for the presence of $\mathrm{C}=\mathrm{O}$ group in IR spectra. In addition, ${ }^{1} \mathrm{H}$ NMR spectrum clearly shows the loss of $\mathrm{H}_{\text {methylidene }}$ leading to compounds $\mathbf{4 a - j}$. In the present work the formulas of compounds $\mathbf{3 a}-\mathbf{j}, \mathbf{4 a - j}$ were found by elemental analysis and their structures were determined by IR, ${ }^{1} \mathrm{H}-\mathrm{NMR}$ and ${ }^{13} \mathrm{C}-\mathrm{NMR}$ spectra data.
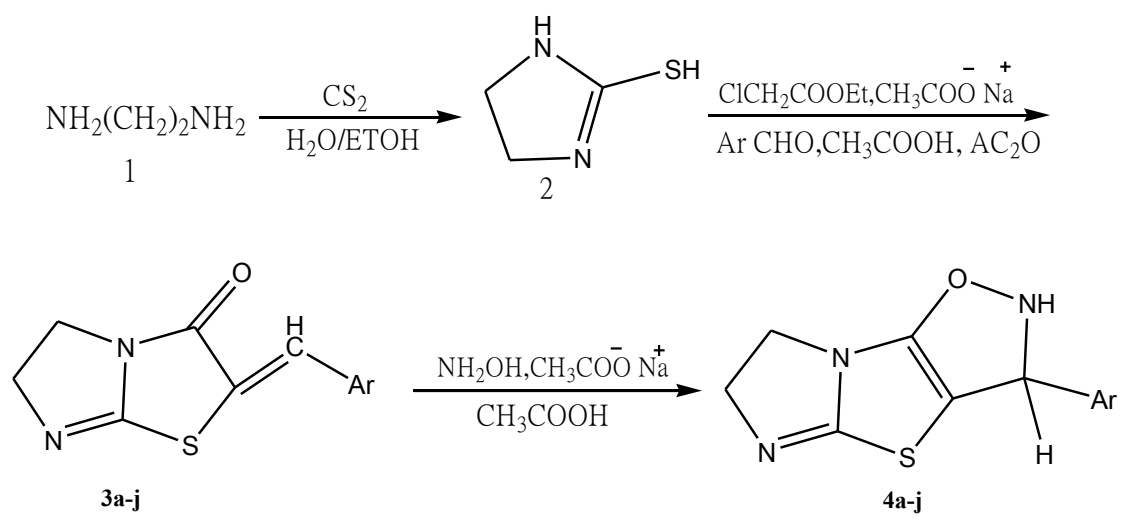

Ar, 3a, 4a: $\mathrm{C}_{6} \mathrm{H}_{5}, \mathbf{3 b}, \mathbf{4 b}: 2-\mathrm{OHC}_{6} \mathrm{H}_{4}, \mathbf{3 c}, \mathbf{4 c}: 4-\mathrm{CH}_{3} \mathrm{C}_{6} \mathrm{H}_{4}, \mathbf{3 d}, \mathbf{4 d}: 3-\mathrm{OCH}_{3} \mathrm{C}_{6} \mathrm{H}_{4}, \mathbf{3 e}, \mathbf{4 e}: 3-\mathrm{BrC}_{6} \mathrm{H}_{4}, \mathbf{3 f}$, 4f: 3-ClC $\mathrm{H}_{4}, \mathbf{3 g}, \mathbf{4 g}$ : 2- $\mathrm{NO}_{3} \mathrm{C}_{6} \mathrm{H}_{4} ; \mathbf{3 h}, \mathbf{4 h}: 3-\mathrm{NO}_{2} \mathrm{C}_{6} \mathrm{H}_{4}, \mathbf{3 i}, \mathbf{4 i}: 4-\mathrm{NO}_{2} \mathrm{C}_{6} \mathrm{H}_{4}, \mathbf{3 j}, \mathbf{4 j}: \mathrm{C}_{4} \mathrm{H}_{3} \mathrm{O}$

Figure 1. Synthetic pathway for preparation of $\mathbf{4 a - j}$.

The IR data were very informative and provided evidence for the formation of the expected structures. The compounds were isolated in satisfactory yields (50-87\%) and purified by crystallization, using from appropriate solvent. In the IR spectra of $\mathbf{3 a - j}$ and 4a-j showed an absorption bond at $1214-1231 \mathrm{~cm}^{-1}$ for $\mathrm{C}-\mathrm{N}, 3150-3185 \mathrm{~cm}^{-1}$ due to $\mathrm{NH}$ and $1698-1713 \mathrm{~cm}^{-1}$ characteristic $(\mathrm{C}=\mathrm{O})$ of $\alpha, \beta$-unsaturated ketone [this shift to lower frequency is due to conjugation with exocyclic double bond]. Also, the ${ }^{1} \mathrm{H}$ NMR spectrum in DMSO- $\mathrm{d}_{6}$ and $\mathrm{CDCl}_{3}$ of compounds $\mathbf{3} \mathbf{a}-\mathbf{j}$ and $\mathbf{4} \mathbf{a}-\mathbf{j}$ is in agreement with its structure, which revealed at $3.52-3.88 \mathrm{ppm}$ due to aliphatic proton and all the aromatic protons were observed at expected regions. The signal of NH groups appears as a broad signal in the same range as those of the aromatic protons and $\mathrm{OH}$ proton was observed as a broad at $9.50 \mathrm{ppm}$.

\section{Conclusion}

In conclusion, we have presented a facile route for the synthesis of new imidazo $[2,1-b][1,3]$ thiazole derivatives using 4, 5-dihydro- $1 H$-imidazol-2-thiol, This compound was used as a key compound for this study and for further syntheses of other fused heterocyclic. Compound 2 was treated with ethyl chloroacetate and benzaldehyde derivatives to afford compound 3a-j. These compounds were used to synthesize a series of new other compounds $4 \mathbf{a}-\mathbf{j}$ by condensation reaction with hydroxylamine hydrochloride. Because of the compounds $\mathbf{4 a - j}$ having $-\mathrm{NH}$ - factors can be used for the synthesis of newer compounds. As it is mentioned above probably all new synthesized compounds have Pharmacology activities. 


\section{References}

1. Vaghasia S L and Shah V H, J Serb Chem Soc., 2007, 72, 109

2. Lednicer D and Mitscher L A, In Organic Chemistry of Drug Synthesis, Wiley Interscienc New York, 1997.

3. Andreani A, Granaiola M, Leoni A, Locatelli A, Morigi R, Rambaldi M, Lenaz G, Fato R, Bergamini C and Farruggia G, J Med Chem., 2005, 48, 3085

4. Robert J F, Boukraa S, Panouse J J, Loppinet V and Chaumont J P, Eur J Med Chem., 1990, 25, 731.

5. Andreani A, Rambaldi M, Leoni A, Morigi M, Locatelli A, Georgi G, Lenaz G, Ghelli A and Esposti M D, Eur J Med Chem, 1999, 34, 888

6. Adams J L, Boehm J C, Gallagher T F, Kassis S, Webb E F, Sorenson M, Garigipati R, Griswold D E and Lee J C, Bioorg Med ChemLett 2001, 11, 2867.

7. Nakamura T, Kakinuma H, Umemiya H, Amada H, Miyata N, Taniguchi K, Bando K and Sato M, Bioorg Med Chem Lett.. 2004, 14, 333.

8. Venkatesan A M, Agarwal A, Abe T, Ushirogochi H O, Santos D, Li Z, Francisco G, Lin Y I, Peterson P J, Yang Y, Weiss W J, Shales D M and Mansour T S, Bioorg Med Chem., 2008, 16, 1890.

9. Bhandari K, Srinivas N, Keshava G B S and Shukla P K, Eur J Med Chem., 2011, in press.

10. Congiu C, Cocco M T and Onnis V, Bioorg Med Chem Lett., 2008, 18, 989.

11. Han M and Kim D H, BioorgMed Chem Lett., 2001, 11, 1425

12. Khazi I M and Koti R S, Indian J Chem., 2004, 43B, 393.

13. Mulwad V V and Shirodkar J M, Indian J Heterocycl Chem., 2002, 11, 291.

14. Gian C L, Fracesco P, Gian G G, "Antibiotics - A Multidisciplinary approach", $2^{\text {nd }}$ Edn., Plenum Press, New York, 1995, 16-19.

15. Mohamed S F, Thabet H K, Mostafa E E, Abdalla M M and Shafik S H, Synth World J Chem., 2009, 4(2),100.

16. Hayam H, Shamroukh A H and Rashad A E, Acta Pharm., 2006, 56, 231.

17. Bhaskar V H, Kumar M, Sangameswaran B and Balakrishnan B R, Rasayan J Chem., 2008, 1, 218.

18. Al-Rashood K A and Abdel-Aziz H A, Molecules, 2010, 15, 3775. 


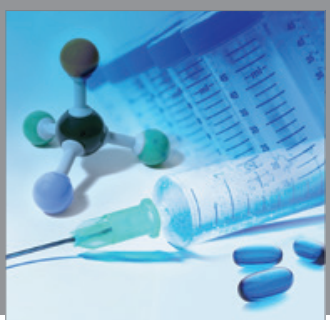

International Journal of

Medicinal Chemistry

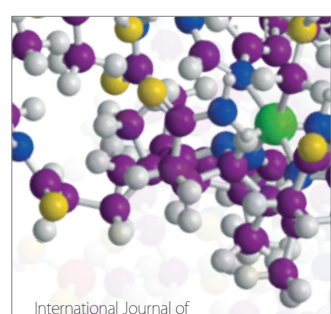

Carbohydrate Chemistry

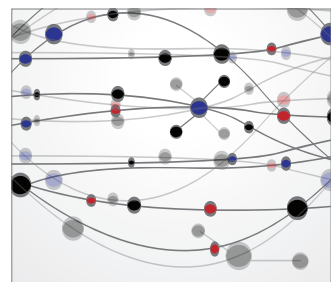

The Scientific World Journal
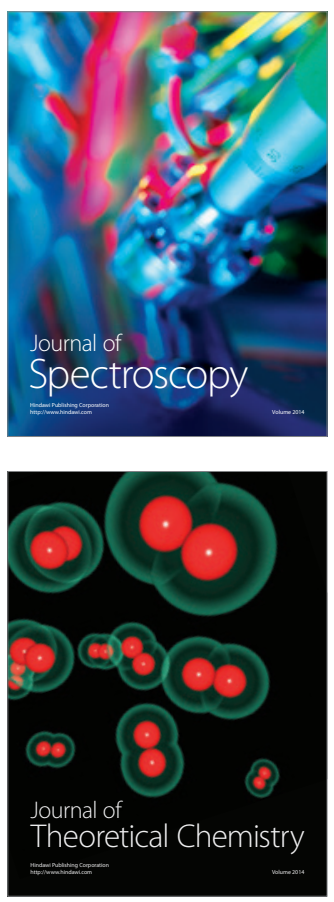
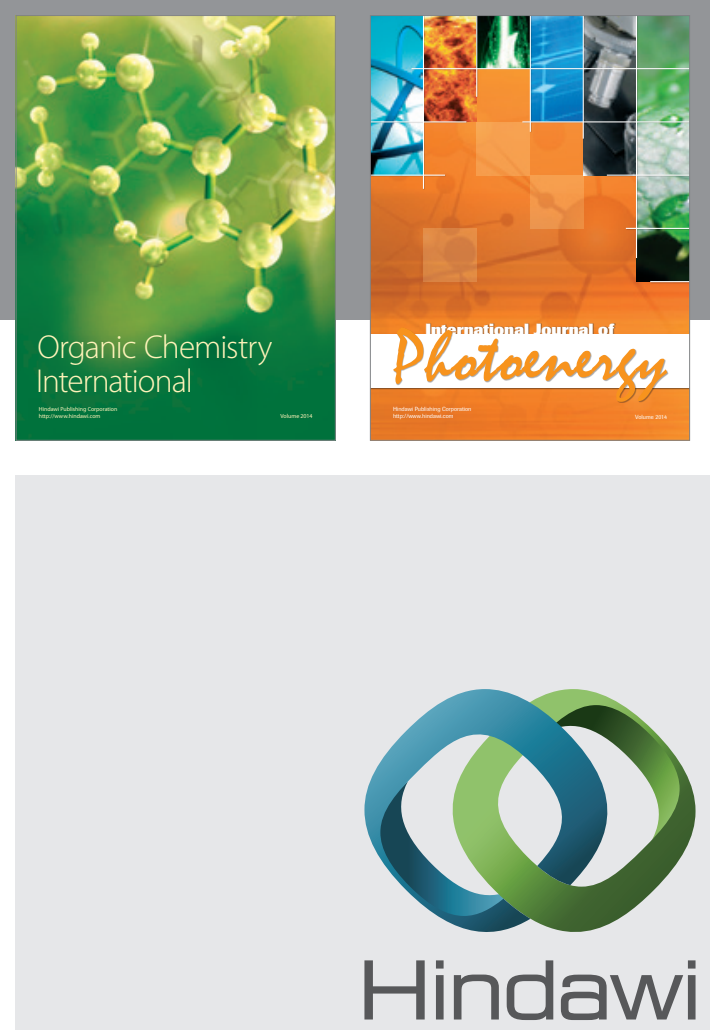

Submit your manuscripts at

http://www.hindawi.com
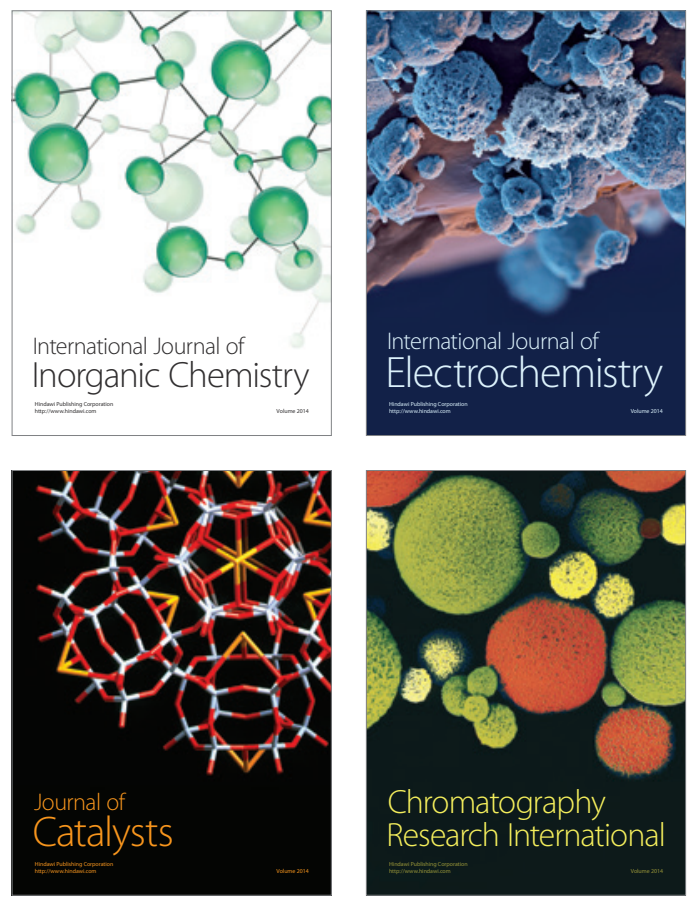
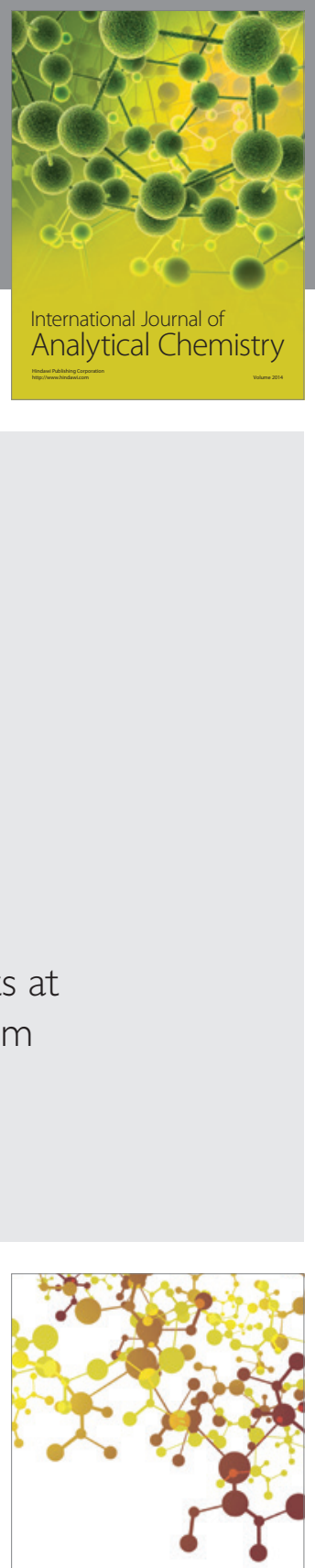

Journal of

Applied Chemistry
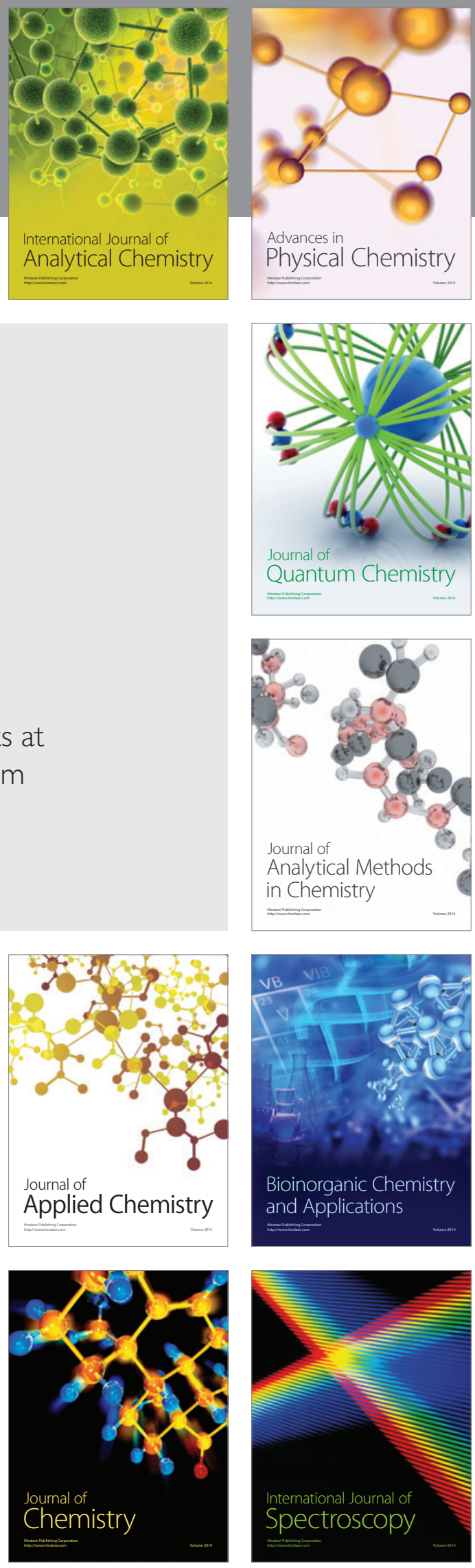